\title{
Lateralisation of cortical function during cognitive tasks: regional cerebral blood flow studies of normal individuals and patients with schizophrenia
}

\author{
Karen Faith Berman, Daniel R Weinberger
}

\begin{abstract}
To assess cognitively-related regional asymmetries of brain function, regional cerebral blood flow (rCBF) was determined by the xenon ${ }^{133}$ inhalation method while normal subjects performed 10 different tasks and also while they were at rest. In addition to healthy subjects, patients with schizophrenia were also studied. A total of 447 rCBF studies were carried out during the following conditions: the Wisconsin Card Sort Test, a numbers matching test, a symbols matching test, Raven's Progressive Matrices, an auditory discrimination test, an auditory control task, two versions of a visual continuous performance task, line orientation, semantic classification, and resting. On the whole, those tasks that seem to require or allow for internal verbalisation resulted in the greatest activation of the left hemisphere compared with the right; right hemisphere activation predominated only in the two tasks primarily involving attention and vigilance. Furthermore, a consistent regional topography of normal cerebral functional laterality was seen: under most conditions left prefrontal cortical activity exceeded that of right prefrontal cortex; during all non-auditory tasks, parieto-occipital cortical activity had an opposite pattern-greater right than left. During most conditions the schizophrenic patients displayed the same pattern. While several cognitively specific between-group differences were found, no single cortical region was consistently implicated and no specific direction of abnormal asymmetry predominated. These data suggest that there is a predominant task-independent functional pattern of cortical activity emphasising relatively greater left anterior and right posterior activation. This pattern may reflect the verbal and attentional primacy of these areas, respectively.
\end{abstract}

The concept that the left and right cerebral hemispheres differ anatomically and functionally has been an important focal point in the understanding of the human brain for over a century. ${ }^{1}$ Early investigations of hemispheric asymmetry were pioneered in 1861 and 1865 by $\mathrm{Broca}^{2}$ who proposed a locus for language in the left hemisphere. Recent studies have demonstrated differential behavioural effects of lateralised brain lesions as well as differences in performance of the right and left hemispheres of commissurotomised patients on cognitive tasks. ${ }^{34}$

In the normal brain hemispheric specialisation has been inferred from a diverse body of experimental evidence. Indirect indicators of hemispheric function have been studied, including lateralisation of perceptual advantages and asymmetries of motor, autonomic and cognitive responses. New techniques for studying brain morphology and physiology in living patients provide more direct approaches to defining the normal topography of structural and functional lateralisation, potential disturbances of lateralisation and the neural networks underlying them. Anatomical differences between the two hemispheres have been demonstrated with radiographic computerised tomography (CT) ${ }^{56}$ and with magnetic resonance imaging (MRI). ${ }^{7}$ Furthermore, measurements of normal brain function in vivo using electrophysiological and regional cerebral blood flow (rCBF) techniques have confirmed that the two hemispheres are differentially active in response to the demands of different behavioural tasks. ${ }^{8-10}$ Few studies, however, have systematically examined the regional topography and variability of these hemispheric differences across a variety of simple and complex tasks.

Alterations in lateralised brain organisation have also been hypothesised to play a role in a number of neurological and psychiatric disorders involving disturbances of higher cognitive function, ${ }^{11} 12$ including dyslexia, Pick's disease and Alzheimer's disease, ${ }^{11}$ as well as autism ${ }^{13}$ and depression. ${ }^{14}$ Schizophrenia, in particular, is a disorder in which a primary disturbance of-laterality has been postulated and extensively studied. ${ }^{14-16}$ For this reason, patients with this illness represent a potentially interesting comparison group for the study of normal cerebral lateralisation.

Observations of disturbed verbal behaviour in schizophrenia ${ }^{17}$ and the occurrence of schizophrenia-like psychoses in patients with left hemisphere lesions, ${ }^{18}$ notably temporal lobe epileptic foci, ${ }^{19}$ have been taken by some to suggest greater left than right hemisphere dysfunction in schizophrenia. Opinions have differed as to whether this putative left hemisphere dysfunction represents left overactivation, ${ }^{20}$ left hemisphere compensation for right hemisphere deficit, ${ }^{21}$ left hypofunction, or disordered interhemispheric communication. ${ }^{223}$ Others have concentrated on the 
right hemisphere as the primary $\operatorname{site}^{2425}$ or on theories of bilateral dysfunction. ${ }^{26}$ Regardless of the hemisphere implicated, a wide variety of experimental approaches, including tachistoscopic presentation of visual stimuli to a specific hemisphere ${ }^{22}$ and dichotic listening tasks, ${ }^{27}$ as well as examination of conjugate lateral eye movements, ${ }^{20}$ skin conductance ${ }^{28} 29$ and neuropsychological deficits, ${ }^{143031}$ have been used to search for altered lateralisation in schizophrenia. Although a sizeable body of literature has ensued (reviewed in refs 15 and 32), no cohesive pattern of findings has emerged.

Brain imaging techniques have also been applied to the study of schizophrenia. While most in vivo morphometric investigations of this illness have focused on bilaterally represented structural elements such as the lateral ventricles or cortical sulci, some studies of brain structure that are consistent with the notion of altered hemispheric differentiation have been reported. ${ }^{33}$ However, no consistent anatomical asymmetries have been found. ${ }^{34}$

Similarly, direct measurements of brain function in schizophrenia have not yielded conclusive answers. For example, many electroencephalography (EEG) and evoked potential studies have reported abnormal asymmetries of brain electrical activity, ${ }^{35}$ but the results have been inconsistent. Some show right hemisphere dysfunction to be more common in patients, ${ }^{25}$ while others indicate more abnormalities on the left. ${ }^{36-38}$ Similar disparities are reflected in $\mathrm{rCBF}^{39-41}$ and positron emission tomography (PET) studies $^{4243}$ of brain activity. In many such investigations the effects of experiential epiphenomena, which can significantly contaminate studies of brain function, have not been controlled for or considered in the interpretation of results.

Thus, despite compelling theoretical arguments about disordered laterality as applied to schizophrenia and despite the publication of well over 70 studies exploring the possibility of abnormal cerebral lateralisation in schizophrenia, ${ }^{32}$ no clear consensus has been reached. This paper used one functional brain imaging technique, xenon ${ }^{133}$ inhalation $\mathrm{rCBF}$, to explore lateralisation of cortical function during a variety of cognitive tasks and states in normal individuals and in patients with schizophrenia.

\section{Methods}

Subjects The patient and normal control groups who participated in these 11 cognitive activation rCBF studies are described in table 1. Nine of the 11 studies included both a schizophrenic patient group and a healthy control group. For the Line Orientation and Semantic Classification studies the patient group consisted of dyslexics; the data for the dyslexics have been presented elsewhere, ${ }^{44}$ and in the present paper only the results for normal subjects for these two tasks will be considered. Patients and their respective control groups did not differ in age, sex or hand preference as determined by the laterality index of the Halstead-Reitan Battery (table 1).

Each subject signed consent in accordance with the Institutional Review . Board and Radiation Safety Committee requirements. All patients were housed on National Institute of Mental Health (NIMH) research wards at the NIMH Neurosciences Center of St Elizabeths, Washington, DC, or the National Institutes at the Health Clinical Center, Bethesda, Maryland. NIHM research patients tend to be moderately to severely ill, to have incomplete response to treatment and to require frequent admission to hospital. All patients in the current study fulfilled DSMIII-R criteria for chronic schizophrenia and all patients and normal subjects who had major medical problems were excluded. Control subjects were recruited from hospital staff and respondents to advertisements in the National Institutes of Health volunteer registry. They were screened for history of major psychiatric disorders, neurological disorders and drug abuse. Subjects were taking no medications that might affect rCBF at the time of the study. Furthermore, patients had been free of neuroleptic and all other medications for at least four weeks at the time of the study. Subjects were asked to refrain from nicotine and caffeine before the studies.

$r C B F$ Measurements The xenon ${ }^{133}$ inhalation technique described by Obrist and colleagues ${ }^{45-47}$ and modified by Deshmuhk and Meyer $^{48}$ and Risberg and colleagues ${ }^{949}$ was used to measure rCBF during eleven different cognitive conditions described below. We have instituted several further changes in the technique as described elsewhere. ${ }^{50-52}$ In brief, tracer concentrations of the inert gas xenon ${ }^{133}$

Table 1 Subject demographics

\begin{tabular}{|c|c|c|c|c|c|c|c|c|c|c|}
\hline \multirow[b]{2}{*}{ Testing condition } & \multicolumn{2}{|c|}{ Sample size } & \multicolumn{4}{|c|}{ Age (SEM) } & \multicolumn{2}{|c|}{$\operatorname{Sex}(F / M)$} & \multicolumn{2}{|c|}{ Hand preference $(R / L)$} \\
\hline & $n m l$ & sch & $n m l$ & & sch & & $n m l$ & sch & $n m l$ & sch \\
\hline $\begin{array}{l}\text { Resting } \\
\text { Wisconsin Card Sort } \\
\text { Numbers Matching } \\
\text { CPT-X } \\
\text { CPT-AX } \\
\text { Raven's Matrices } \\
\text { Symbols Matching } \\
\text { Auditory Discrimination } \\
\text { Auditory Control Task } \\
\text { Line Orientation } \\
\text { Semantic Classification }\end{array}$ & $\begin{array}{l}50 \\
25 \\
25 \\
17 \\
17 \\
25 \\
25 \\
18 \\
18 \\
14 \\
14\end{array}$ & $\begin{array}{l}31 \\
30 \\
30 \\
18 \\
18 \\
24 \\
24 \\
12 \\
12 \\
-\end{array}$ & $\begin{array}{l}26 \cdot 9 \\
30 \cdot 6 \\
30 \cdot 6 \\
28 \cdot 4 \\
28 \cdot 4 \\
28 \cdot 2 \\
28 \cdot 2 \\
29 \cdot 8 \\
29 \cdot 8 \\
22 \cdot 8 \\
22 \cdot 8\end{array}$ & $\begin{array}{l}(0 \cdot 9) \\
(1 \cdot 6) \\
(1 \cdot 6) \\
(2 \cdot 1) \\
(2 \cdot 1) \\
(0 \cdot 9) \\
(0 \cdot 9) \\
(1.9) \\
(1.9) \\
(0 \cdot 8) \\
(0.8)\end{array}$ & $\begin{array}{l}27 \cdot 9 \\
29 \cdot 0 \\
29 \cdot 0 \\
29 \cdot 5 \\
29 \cdot 5 \\
28 \cdot 5 \\
28 \cdot 5 \\
30 \cdot 1 \\
30 \cdot 1 \\
=\end{array}$ & $\begin{array}{l}(1.3) \\
(1.3) \\
(1.3) \\
(1.9) \\
(1.9) \\
(1.0) \\
(1.0) \\
(1.6) \\
(1.6)\end{array}$ & $\begin{array}{l}15 / 35 \\
9 / 16 \\
9 / 16 \\
7 / 10 \\
7 / 10 \\
7 / 18 \\
7 / 18 \\
3 / 9 \\
3 / 9 \\
0 / 14 \\
0 / 14\end{array}$ & $\begin{array}{l}9 / 22 \\
6 / 24 \\
6 / 24 \\
6 / 12 \\
6 / 12 \\
6 / 18 \\
6 / 18 \\
8 / 10 \\
8 / 10 \\
=\end{array}$ & $\begin{array}{l}43 / 7 \\
21 / 4 \\
21 / 4 \\
15 / 2 \\
15 / 2 \\
22 / 3 \\
22 / 3 \\
16 / 2 \\
16 / 2 \\
14 / 0 \\
14 / 0\end{array}$ & $\begin{array}{l}26 / 3 \\
26 / 4 \\
26 / 4 \\
16 / 2 \\
16 / 2 \\
23 / 1 \\
23 / 1 \\
12 / 0 \\
12 / 0 \\
=\end{array}$ \\
\hline
\end{tabular}


were inhaled by each subject for one minute and the rate of elimination of xenon ${ }^{133}$ gamma rays from 32 cortical regions was monitored extracranially with 32 scintillation detectors over the next 11-14 minutes. Grey-matter rCBF was calculated as the initial slope (IS) of each clearance curve. This parameter has been defined and validated as a measure of greymatter rCBF by Obrist and Wilkinson. ${ }^{53}$

rCBF Testing Conditions Each subject had three consecutive rCBF measurements separated by a period of at least 30 minutes and carried out under three different testing conditions. The three measurements were performed in either a single morning or afternoon. Each subject's first rCBF determination was made during a "resting" condition. Following the initial resting state $\mathrm{rCBF}$ determination, the second and third procedures were carried out during two different cognitive activation conditions. On a single day each subject performed one of the following pairs of tasks: pair 1: the Wisconsin Card Sort (WCS) and the Numbers Match (NM); pair 2: two versions of a visual Continuous Performance Task (CPT); pair 3: Raven's Progressive Matrices and the Symbols Matching Task; pair 4: Auditory Discrimination and the Auditory Control task; or pair 5: Line Orientation and Semantic Classification. The sequence in which activation tasks were presented within each pair was counterbalanced across subjects to control for the possibility of an order effect. ${ }^{545}$ The resting state condition and 10 cognitive activation tasks are described below. The results of nonlateralised analyses for some of the subjects have been previously reported. ${ }^{44} 51$ 56-58

A two to four minute period preceded each rCBF measurement during which a reading of background radiation was made and this allowed subjects to adapt to the rCBF laboratory environment. Resting conditions or activation paradigms began approximately one minute before the xenon ${ }^{133}$ inhalation and were continued throughout the entire 12 to 15 minute rCBF measurement period. Sensory and motor events extraneous to the testing condition being studied were kept to a minimum.

Resting condition Subjects were asked to remain awake and motionless with eyes closed. Otherwise, this procedure involved no prescribed sensory, cognitive, or motor events. Resting studies reported here always constituted a subject's first exposure to rCBF measurements.

Wisconsin Card Sort The Wisconsin Card Sort (WCS) is a problem-solving, abstract reasoning test validated by Milner $^{5060}$ as a sensitive indicator of frontal lobe integrity in man. The WCS is particularly sensitive to dysfunction of dorsolateral prefrontal cortex, ${ }^{61}$ and patients with schizophrenia, as well as those with dorsolateral prefrontal lesions, do poorly on this test. ${ }^{51596062} \mathrm{~A}$ semi-automated version of this task was developed for use during $\mathrm{rCBF}$ procedures. ${ }^{51}$
Numbers Match (NM) For the NM, subjects were shown slides of the numbers one to four which appeared in random order and which they were asked simply to match with one of four similarly numbered switches.

Continuous Performance Task-X (CPT$X)$ The CPT is a test of sustained attention and vigilance. ${ }^{63}$ For our visual versions of this task, ${ }^{56}$ letters were flashed rapidly on a screen one at a time during the rCBF measurement. The subject was instructed to press a button, which was held in the right hand, as quickly as possible upon the appearance of the letter "X." If the subject responded quickly enough, a green reinforcement light showed and the response was scored as correct.

Continuous Performance Task-AX (CPT$A X)$ During this version of the CPT subjects were asked to respond only to those "X's" which followed an "A." The CPT-AX was "dynamically paced" (that is, correct responses resulted in shorter stimulus durations, interstimulus intervals and available response times, allowing performance to be optimised over a wide range of ability levels). To ensure that subjects comprehended the instructions, short trial runs of each CPT were given before the rCBF procedure itself.

Raven's Progressive Matrices (RPM) This abstract reasoning task, first described in $1938,{ }^{64}$ has been shown to correlate with formal measures of IQ.$^{65}$ During our automated version of this task subjects were shown slides, each consisting of a pattern, or "matrix," with a missing piece. The task was to choose one of eight possible pieces to correctly complete the pattern.

Symbols Matching Task This test was designed as a control task for the RPM paradigm. Subjects were shown slides containing a test design (one of the stimuli from the RPM) and eight answer designs (also chosen from RPM stimuli), one of which was identical to the test design. The task was simply to match the test design to the identical answer design by pressing one of eight switches corresponding to the eight possible answers.

Auditory Discrimination With eyes closed and patched, subjects listened to a tape on which were recorded a series of pairs of sounds which were either identical or slightly different. Subjects were asked to press one of two buttons after each sound pair-button 1 if the two sounds were the same, or button 2 if the two sounds were different. Before the rCBF procedure subjects were trained to criterion in associating the correct meaning to each button. Some sound pairs were moderately complex monotonal rhythm patterns taken from the Seashore Rhythm Test, and some were nonsense syllables taken from the Speech Sound Perception Test. ${ }^{66}$

Auditory Control Task For this task subjects listened to the same tape as for the 
Auditory Discrimination task. However, they were not asked to determine whether the sound pairs were the same or different, but only to press button 1 after each pair of sounds to indicate that they had heard the sounds.

Semantic classification For each trial of this task subjects were shown two slides. First, the stimulus slide consisted of a single word. This was followed by an answer slide on which was listed four numbered choices-1 Food, 2 Body Part, 3 Animal, 4 Tool-from which subjects were asked to choose the category to which the stimulus word belonged.

Line orientation Benton's Judgement of Line Orientation test, which is particularly sensitive to right hemisphere lesions, ${ }^{51}$ was adapted for this task. Each item consisted of two slides: a stimulus slide showing two angled lines and a response slide with a protractor-like arrangement of lines from which the subject was asked to choose the line corresponding to the angle of the stimulus slide.

\section{Statistical analysis}

Statistical analyses were performed on rCBF laterality indices derived in the following manner. For each task the 32 grey-matter rCBF values for each subject were collapsed into 10 cortical regions (five per hemisphere): left and right prefrontal, precentral, temporal, parietal and parieto-occipital. ${ }^{51}$ This approach reduced the possibility of falsely positive findings in the face of many statistical comparisons. Each regional $\mathrm{rCBF}$ value was calculated as the mean of the individual values comprising it. These regional values were then used to generate the laterality indices (as a percentage) by subtracting each right region from the homologous left region and dividing the difference by the mean of the left and right regions: $[\mathrm{L}-\mathrm{R} /((\mathrm{R}+\mathrm{L}) /$ $2)]^{\star} 100$. One advantage of the laterality index is that it is independent of blood carbon dioxide tension which has been shown to directly affect absolute rCBF ${ }^{68-70}$ and is not affected by other potential $\mathrm{rCBF}$ artifacts (for example individual differences in partition coefficients, respiratory efficiency, etc).

Within-group comparisons of regional and hemispheric laterality indices To test for significant left-right differences within each subject group for a given task, we used Hotelling's $\mathrm{T}^{2}$ test, a multivariate $T$ statistic that can be applied to repeated measures and/or paired data. ${ }^{71}$ This statistic evaluates the null hypothesis that the means of the multiple variables (that is, the five regional laterality indices) do not differ, and in this case that they do not differ from zero. Thus, if the left and right rCBF values for each region were similar, then each laterality index would approach zero, and Hotelling's $T^{2}$ would not be significant. Post hoc individual univariate analyses testing whether each regional index differed from zero were also carried out. Similarly, the difference between relative mean left and right hemisphere rCBF (as a percentage of whole brain-blood flow) for each task was also tested. Within the limits of sensitivity and resolution of the xenon $^{133}$ rCBF technique, these analyses examine whether significant physiological lateralisation occurred during any of the tasks.

Between-groups comparisons of hemispheric and regional laterality indices To test for mean hemispheric laterality differences between the groups, mean hemispheric laterality indices were computed analogously to the regional laterality indices: [(left hemisphere-right hemisphere)/whole brain] ${ }^{\star} 100$. ANOVA was used to compare hemispheric laterality indices across groups for each task.

To test for overall regional laterality differences between the groups for each task, the five regional laterality indices were analysed with multivariate analysis of variance (MANOVA), a statistical approach that simultaneously accounts for the effects of all five regions and minimises Type 1 statistical error (that is, false positive results). This was followed by univariate analysis of variance (ANOVA) for each regional laterality index alone, a statistical approach that minimises Type 2 error (that is, falsely negative results).

\section{Results}

Laterality within each group

Hemispheric findings As seen in fig 1 , both groups tended to have relatively higher left than right hemisphere blood flow in most conditions. The left-right hemispheric difference reached statistical significance for the Raven's and Symbols tasks in both groups and for the auditory discrimination task in the normal controls. In contrast, the only tendency for mean right hemisphere blood flow to exceed that of the left hemisphere was noted in normal subjects during both versions of the CPT ( $p=$ 0.065 for CPT-X; $p<0.2$ for CPT-AX).

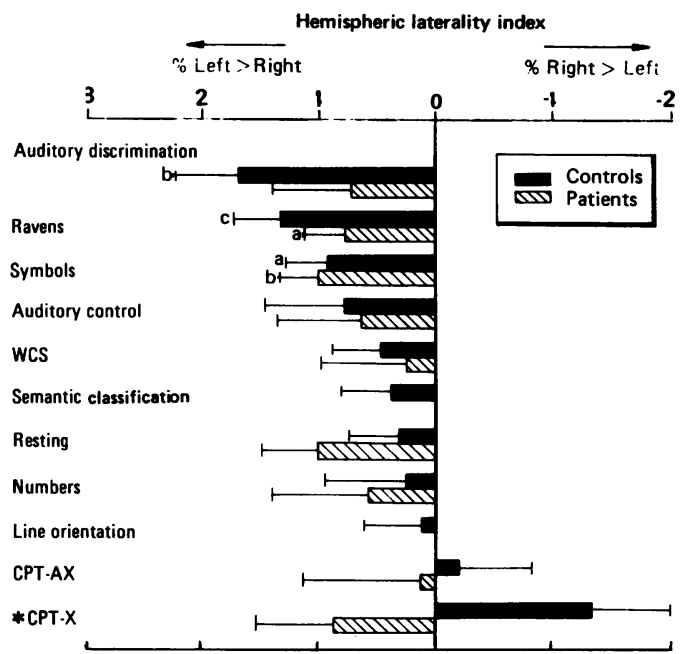

Figure 1 Hemispheric laterality indices for normal subjects and patients with schizophrenia. Sample sizes are given in table 1. Values were calculated as follows:

(left hemisphere-right hemisphere) entire brain

$a=P \leqslant 0.05$ for within-group comparison $b=P \leqslant 0.01$ for within-group comparison $c=P \leqslant 0.005$ for within-group comparison $d=P \leqslant 0.001$ for within-group comparison $\star=P \leqslant 0.05$ for between-group comparison Bars represent standard error. 
Table 2 Results of within-group regional laterality statistical analysis

Normal subjects

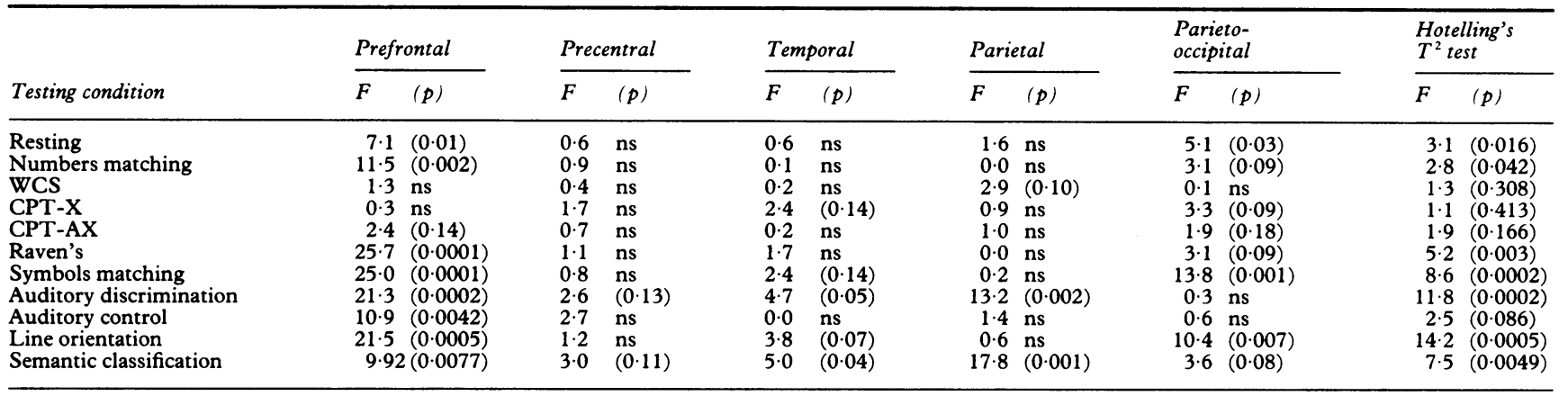

Patients

\begin{tabular}{|c|c|c|c|c|c|c|c|c|c|c|c|c|}
\hline \multirow[b]{2}{*}{ Testing condition } & \multicolumn{2}{|c|}{ Prefrontal } & \multicolumn{2}{|c|}{ Precentral } & \multicolumn{2}{|c|}{ Temporal } & \multicolumn{2}{|c|}{ Parietal } & \multicolumn{2}{|c|}{$\begin{array}{l}\text { Parieto- } \\
\text { occipital }\end{array}$} & \multicolumn{2}{|c|}{$\begin{array}{l}\text { Hotelling's } \\
T^{2} \text { test }\end{array}$} \\
\hline & $F$ & $(p)$ & $F$ & $(p)$ & $F$ & $(p)$ & $F$ & $(p)$ & $F$ & $(p)$ & $F$ & (p) \\
\hline Resting & $15 \cdot 0$ & $(0.0005)$ & 0.7 & ns & 0.6 & ns & 0.5 & ns & $0 \cdot 2$ & ns & $4 \cdot 2$ & $(0.006)$ \\
\hline Numbers matching & $6 \cdot 2$ & $(0.02)$ & 0.0 & ns & 0.4 & ns & $0 \cdot 3$ & ns & $16 \cdot 7$ & $(0.0003)$ & $5 \cdot 6$ & $(0.001)$ \\
\hline WCS & $4 \cdot 6$ & $(0.04)$ & 0.0 & ns & 0.7 & ns & $0 \cdot 2$ & ns & $1 \cdot 2$ & ns & $2 \cdot 1$ & $(0 \cdot 104)$ \\
\hline CPT-X & $4 \cdot 4$ & $(0.05)$ & 0.0 & ns & 0.0 & ns & $2 \cdot 3$ & $(0 \cdot 15)$ & $0 \cdot 0$ & ns & $1 \cdot 3$ & $(0.314)$ \\
\hline CPT-AX & $0 \cdot 3$ & ns & 0.0 & ns & $0 \cdot 1$ & ns & $2 \cdot 1$ & $(0 \cdot 17)$ & $4 \cdot 2$ & $(0.06)$ & $3 \cdot 3$ & $(0.038)$ \\
\hline Raven's & $21 \cdot 4$ & $(0.0001)$ & $0 \cdot 1$ & ns & $2 \cdot 2$ & $(0 \cdot 15)$ & $3 \cdot 2$ & $(0.09)$ & $0 \cdot 2$ & ns & $11 \cdot 7$ & $(0.0001)$ \\
\hline Symbols matching & $22 \cdot 4$ & $(0.0001)$ & 0.6 & ns & 0.4 & ns & $10 \cdot 7$ & $(0.003)$ & 2.5 & $(0 \cdot 13)$ & $8 \cdot 3$ & $(0.0003)$ \\
\hline Auditory discrimination & $19 \cdot 7$ & $(0.001)$ & $6 \cdot 3$ & $(0 \cdot 03)$ & 0.0 & ns & $3 \cdot 2$ & $(0 \cdot 10)$ & $0 \cdot 3$ & ns & $5 \cdot 0$ & $(0.029)$ \\
\hline Auditory control & $19 \cdot 3$ & $(0.001)$ & $3 \cdot 3$ & $(0 \cdot 10)$ & 0.0 & ns & $1 \cdot 3$ & ns & $0 \cdot 1$ & ns & $13 \cdot 2$ & $(0.002)$ \\
\hline
\end{tabular}

$\mathrm{ns}=\mathrm{p} \geqslant 0 \cdot 2 ; \mathrm{F}$ indicates univariate ANOVA $\mathrm{F}$ for right-left comparison of a particular region.

Figure 2 A-K Regional laterality indices for normal subjects and patients with schizophrenia during 11 different cognitive conditions. Values were calculated as follows:

$$
\frac{\text { left-right }}{(\text { left }+ \text { right }) / 2} \times 100
$$

$A=P \leqslant 0.05$ for withingroup multivariate comparison

$B=P \leqslant 0.01$ for withingroup multivariate

comparison

$C=P \leqslant 0.005$ for

within-group multivariate comparison

$D=P \leqslant 0.001$ for

within-group multivariate comparison

$a=P \leqslant 0.05$ for withingroup univariate regional comparison

$b=P \leqslant 0.01$ for withingroup univariate regional comparison

$c=P \leqslant 0.005$ for withingroup univariate regional comparison

$d=P \leqslant 0.001$ for

within-group univariate regional comparison

$\star=P \leqslant 0.05$ for withingroup univariate regional comparison.
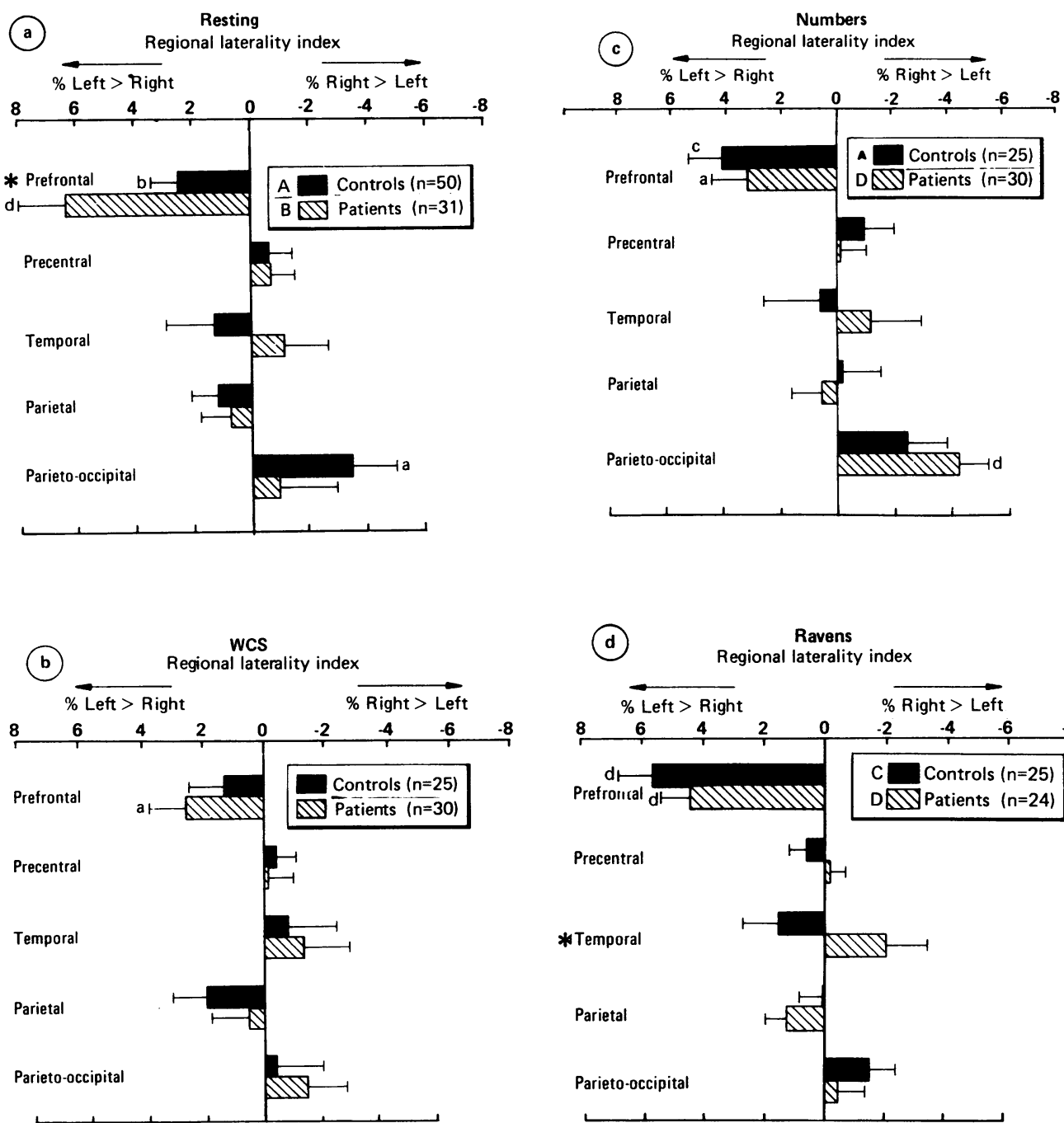

(d)

Ravens
Regional laterality index

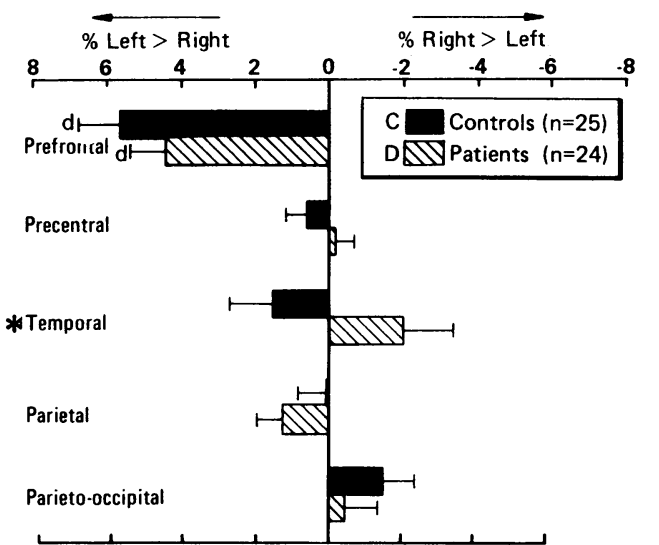



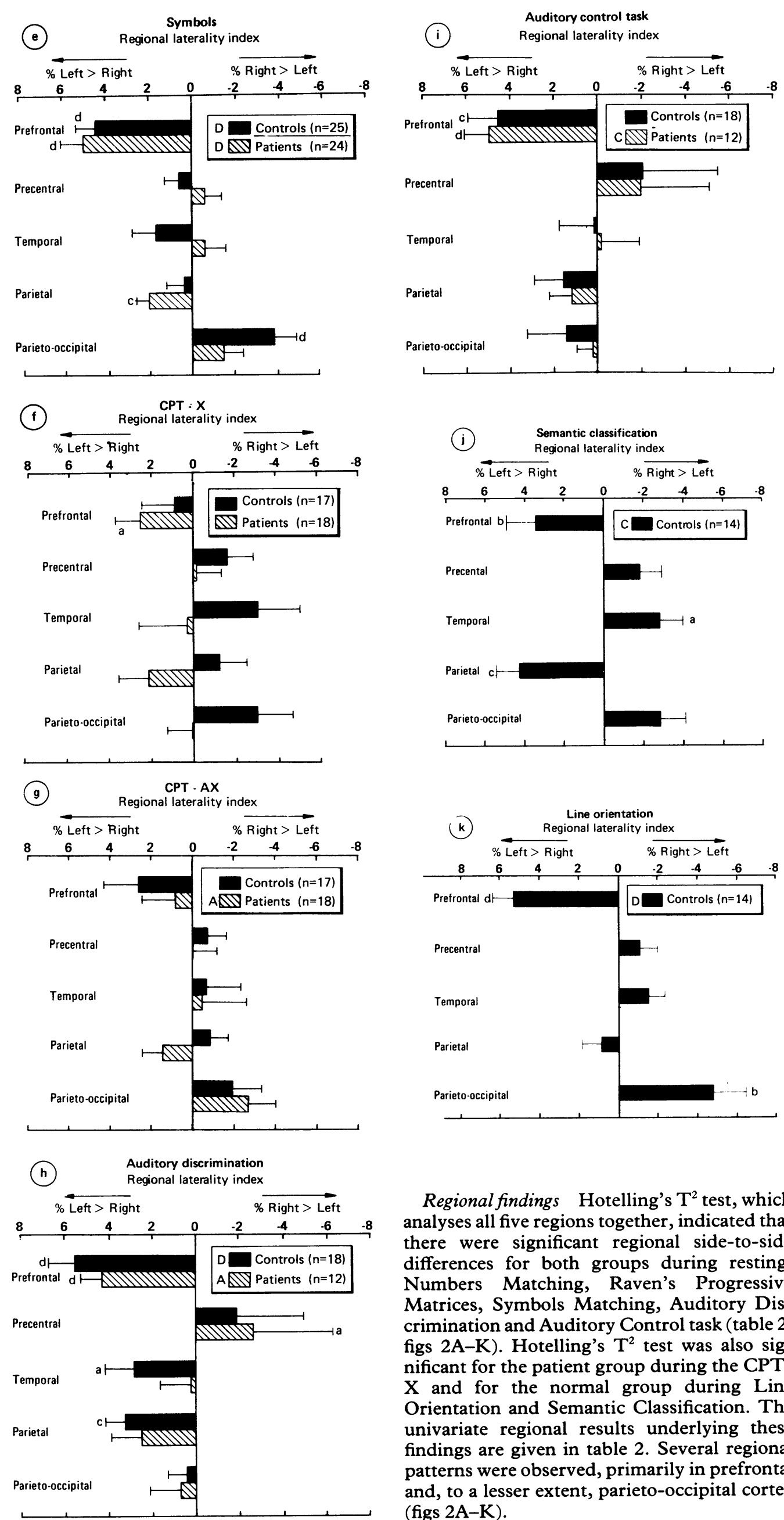

Regional findings Hotelling's $\mathrm{T}^{2}$ test, which analyses all five regions together, indicated that there were significant regional side-to-side differences for both groups during resting, Numbers Matching, Raven's Progressive Matrices, Symbols Matching, Auditory Discrimination and Auditory Control task (table 2, figs $2 \mathrm{~A}-\mathrm{K})$. Hotelling's $\mathrm{T}^{2}$ test was also significant for the patient group during the CPT$X$ and for the normal group during Line Orientation and Semantic Classification. The univariate regional results underlying these findings are given in table 2 . Several regional patterns were observed, primarily in prefrontal and, to a lesser extent, parieto-occipital cortex (figs $2 \mathrm{~A}-\mathrm{K}$ ). 
In all 11 conditions during which normal subjects were tested and all nine conditions during which patients with schizophrenia were tested, both groups showed relatively greater left than right prefrontal rCBF (sign test, $p<$ 0.0001 for both groups). This relative left-right difference was highly significant for the normal control group in eight conditions (all but the WCS, CPT-X and CPT-AX), and in eight of the nine conditions (all but the CPT-AX) for the patient group.

In contrast, left-right differences for parietooccipital rCBF were predominantly opposite in direction to those in the prefrontal region. That is, as seen in figs $2 \mathrm{~A}-\mathrm{K}$, parieto-occipital rCBF tended to be greater on the right than the left in nine of 11 conditions for the control group (all but the two auditory tasks) and in six of nine conditions for the patients (all but the CPT-X and the Auditory Control task in which left and right parieto-occipital rCBF were approximately equal and the Auditory Discrimination Task in which left was slightly but not significantly greater than right).

For regions other than prefrontal and parieto-occipital, no consistent pattern was found and in only a few instances was rCBF lateralised to a significant degree. Lateralisation to the left was seen in parietal cortices of the patients during Symbols Matching, and those of normal subjects during Auditory Discrimination and Semantic Classification. Temporal cortex rCBF of normal subjects lateralised significantly to the left during Auditory Discrimination and to the right during Semantic Classification. Precentral rCBF of the patients lateralised to the right during Auditory Discrimination.

\section{Laterality differences between patients and controls}

Hemispheric findings ANOVAs performed on mean hemispheric laterality indices showed no significant between-group difference for eight of the nine conditions under which both patients and normal controls were studied (fig 1). During the CPT-X, mean hemispheric laterality differed significantly between the two groups. While patients tended to show relatively greater left hemisphere activity, controls had relatively greater mean activity in the right hemisphere.

Regional findings For each of the nine conditions, when patients were compared to normals using MANOVAs in which all five regional laterality indices were examined together, no overall difference between the groups was detected (figs $2 \mathrm{~A}-\mathrm{K}$ ). Consistent with this observation, only one significant individual regional laterality difference between the groups was noted on univariate ANOVAs. During the resting condition, while both groups showed higher left than right prefrontal rCBF values, the degree of left prefrontal lateralisation was significantly greater in the patients $(F=4.85, p=0.03$; fig 2A). Also, during Raven's Matrices a trend toward different temporal lateralisation was noted $(F=3.94, p=0.053)$; normals showed temporal lateralisation to the left, while patients had relatively greater right temporal $\mathrm{rCBF}$ (fig 2D).

\section{Discussion}

Several methodological points will be addressed before discussion of the data. One technical limitation of xenon $^{133}$ inhalation rCBF measurements for studying laterality is "crosstalk." This refers to the possibility that each radiation detector detects gamma rays not only from the immediately underlying cortex, but also from brain tissue directly across the head. In other words, rCBF values measured for one side of the brain may also reflect a small part of the activity of the opposite hemisphere, thereby "washing out" some of the differences between the two hemispheres and rendering the technique less than optimally sensitive to measures of laterality. The effects of crosstalk can be minimised by arranging the detectors radially about the head, ${ }^{73}$ as was done in this study. The degree to which crosstalk remains a limitation is unclear, but the results indicate that the sensitivity of the technique was sufficient to demonstrate a number of highly significant left-right differences within each group. Moreover, a consistent pattern of relatively greater left prefrontal and right posterior activity was found in each subject group. This suggests that, while crosstalk may have diminished the magnitude of the laterality indices, it alone cannot account for the relative paucity and inconsistency of the betweengroup laterality differences delineated above.

Some studies have suggested that men and women may have different cerebral lateralisation and different rCBF patterns. ${ }^{1074}$ Others have reported differences in left- versus righthanded persons. ${ }^{1075}$ In this study the relatively small numbers of women and left-handed subjects precluded their separate analysis. However, the patient and normal groups did not significantly differ in representation of women and left-handers (Fisher exact text, $p$ > $0 \cdot 1$, and the normal subjects for the Line Orientation and Semantic Classification paradigms were all right-handed men. Moreover, additional analyses were performed with the women and non-right handed subjects excluded; while the results reflected the decreased statistical power imposed by the smaller sample size, the basic findings, both within and between groups did not change.

\section{Normal lateralisation of cortical function}

A large body of physiological data attests to the importance and complexity of normal lateralisation of cerebral function. ${ }^{1}$ However, the regional topography of brain functional asymmetry in normal human subjects has been infrequently investigated by direct, noninvasive physiological observations. Early rCBF studies that delineated the normal functional landscape $e^{76}{ }^{77}$ were limited to measuring rCBF of one hemisphere at a time with an invasive intracarotid injection of xenon ${ }^{133}$ dissolved in saline. For example, Ingvar and 
Franzen $^{78}$ studied rCBF during RPM, as we did, but since they were only able to collect data from the dominant hemisphere, they could not examine laterality of function during this task. Roland and colleagues used the same technique to measure rCBF during stereognosis, ${ }^{79}$ auditory and visual discrimination, ${ }^{80} 81$ and attending to a tactile stimulus. $^{82}$ They measured both left and right hemisphere rCBF, but this was done with two separate subject groups. Thus, direct side to side statistical comparisons were not carried out.

Even when methods allowing simultaneous measurements in both hemispheres became available, relatively few investigators specifically reported on normal regional lateralisation. While a variety of activation paradigms have been studied with $\mathrm{rCBF}$, many were not well controlled or standardised; the results of those that were are difficult to directly compare because of differences in the ways that various laboratories have applied the technique. Most studies in which regional data for both hemispheres are available differ in several important methodological aspects, such as different numbers and locations of detectors, a variety of derived blood flow parameters, varying testing and cognitive conditions and different approaches to data analyses.

An additional problem is that many investigators have reported $\mathrm{rCBF}$ activation data in comparison to a resting baseline, which is itself a poorly defined cognitive state with its own variable pattern of lateralisation. We derived hemispheric laterality indices from the published data for a number of resting state rCBF studies carried out by other investigators and found that the results obtained by different laboratories vary widely (from $5 \cdot 1 \%$ to the left to $2.5 \%$ to the right), even for studies all carried out during a "resting state" (analyses available upon request). These discrepancies may reflect methodological variation and/or the poor reliability of in vivo rCBF and brain metabolic data derived during the resting state. ${ }^{83}$ Attempts to systematically delineate a regional topography of functional lateralisation have been severely limited by these factors.

This paper reports the results of 11 different testing conditions carried out in the same laboratory by the same investigators and delineates what appears to be a predominant regional topography of cortical functional laterality. We found that under most conditions, left prefrontal cortical activity exceeds that of right prefrontal cortex. Parieto-occipital cortex, at least during non-auditory tasks, has an opposite pattern - that is, greater right than left activity. It is interesting that these physiological asymmetries contrast with anatomical asymmetries that have been demonstrated at postmortem examination and with CT. In those anatomical studies, frontal lobe has been shown to be longer and wider on the right, while the occipital lobe is longer and wider on the left. ${ }^{1584}$

One explanation for the physiological asymmetries observed in the present studies may be the primacy of left anterior areas in speech and language functions, whether vocalised or in- ternal, and the involvement of right posterior areas in attentional and visuo-spatial processes. Our demonstration of right parieto-occipital predominance during visual tasks agrees with the PET study of Kushner et al ${ }^{85}$ which showed that, during visual stimulation, local cerebral metabolic rates were higher in the right lateral occipital cortex than in the left, regardless of whether the left or right visual hemifield was stimulated. Examination of fig 1 shows that relatively greater mean activity in the whole right hemisphere was seen in normal individuals only during the two tasks that predominantly test attention and vigilance, the CPTs. This observation supports the hypothesis that attentional processes may normally be preferentially mediated by the right hemisphere. ${ }^{86-88}$

In contrast, during the task that involves auditory discrimination of speech sounds and the two tasks that involve complex reasoning (RPM and WCS), the left hemisphere predominated. One clue to the meaning of this latter observation may be offered by the proposal of many investigators that inner speech and thought are related. ${ }^{89}$ It is possible that while reasoning out the complex, abstract problems posed by RPM and the WCS, subjects may "talk to themselves" (subvocalise). Interestingly, Villardita ${ }^{90}$ showed that aphasic patients had the poorest performance on RPM and emphasised "the importance of internal verbalisation processes for a correct choice ...." An alternative possibility is that solving these problems involves verbal/analytic cognitive processing that preferentially activates the left hemisphere.

The CPTs, on the other hand, are not complex tasks, and subjects may not subvocalise during them or use analytic cognitive processes. Indeed, many individuals experience the performance of these tasks as being almost automatic and unconscious. The CPT stimuli are presented at a much faster rate than are the stimuli for the other cognitive tasks employed, perhaps precluding inner speech and/or verbal/analytical cognition. The rapid pace and the need for close attention may favour the right hemisphere. It is interesting that the other five conditions that tended to be left lateralised, the Symbols Matching task, the Auditory Control task, Semantic Classification (itself a verbal task), the Numbers Matching task and the resting state, were not only nondemanding in terms of attention and reasoning, but may have afforded time and opportunity for contemplation, and perhaps analytical thinking if not inner speech. Of the 11 tasks, the Line Orientation task, which is classically associated with right hemisphere function, provoked the greatest degree of right parietooccipital activation, though left and right mean hemispheric rCBF values were nearly equal.

These data also have implications for the interpretation of in vivo cerebral physiological imaging data. The details of what a subject is doing mentally during an $\mathrm{rCBF}$ procedure affect the results, at least in terms of laterality pattern. If he or she is primarily focusing attention or thinking in verbal terms, even if 
the experimental condition itself requires neither, the rCBF pattern may vary to reflect this difference in mental behaviour. The degree to which such "state" variance in rCBF might obscure more important "trait" findings in pathological conditions requires further study.

\section{Lateralisation of cortical function in schizophrenia}

We did not find consistent rCBF laterality differences between patients free from medication with schizophrenia and normal control subjects. Moreover, when conservative multivariate statistical analysis was applied, there were no significant overall laterality differences between the groups; only with a liberal statistical approach (that is, multiple univariate between-group region-by-region comparisons) did any laterality difference emerge. Thus, these data do not lend strong support to the notion of a unitary disorder of cerebral lateralisation underlying schizophrenia. However, the results do not rule out that some subgroup of (or some patients with) schizophrenia may have dysfunction of one hemisphere or the other under some conditions. In fact, several isolated group differences were found in the present studies.

First, during the CPT-X attentional task mean hemispheric rCBF was higher on the right in normal subjects and higher on the left in patients. This may be viewed as consistent with the findings of Gur et al ${ }^{4041}$ which were interpreted as left hemisphere overactivation. However, since the normal response to the CPT-X was shown to involve right hemisphere preponderance, an equally plausible interpretation, that these data represent right hemisphere underactivation in the patients, may also be inferred. Interestingly, attentional deficits or neglect are noted to occur following right-sided lesions of both cortical and subcortical origin ${ }^{9192}$ and, as previously discussed, a role for the right hemisphere in mediating attention has been proposed. ${ }^{86}$ Thus, one possible explanation for (or consequence of) the failure of the patients to activate right hemisphere preferentially during this attentional task may be that they were paying less attention than the control group. Our data suggest a pathophysiological correlate of the attentional deficits commonly seen in schizophrenia ${ }^{93-95}$ and their poor performance on this vigilance test. ${ }^{52}$

A second lateralisation difference between the two groups was noted during the resting condition. During rest, patients showed significantly more left lateralisation of prefrontal rCBF than normal subjects. These data, like the CPT-X results mentioned above, may be viewed as "left overactivation" on the part of the patients, but are just as consistent with right underactivation. Also, since the resting condition has been shown to be particularly difficult to control and standardise between and even within subjects, these findings might reflect physiological concomitants of different subjective states experienced by the two groups. Perhaps the patients talked to them- selves more during the procedure, thereby increasing activity in left hemisphere speech areas. Thus, whether this finding has primary bearing on the illness itself or whether it may be epiphenomenological, remains to be ascertained.

Finally, the third group difference noted was of borderline significance and was opposite in direction to those discussed above. During Raven's Progressive Matrices normal subjects showed lateralisation to the left in temporal cortex, while patients showed greater right temporal $\mathrm{rCBF}$. This result differs from that of Gur et $a l^{4041}$ and emphasises the importance of cognitive state in laterality measurements.

In summary, the diversity of the current findings parallels the lack of consensus in the literature regarding disordered lateralisation in schizophrenia. It also highlights the statedependency of functional laterality. Whether lateralisation is relatively to the right or left, and whether patients and normal subjects differ, depends on what they are doing during the examination and on what brain region one examines. We did not find a consistent disorder of cerebral lateralisation in schizophrenia, and the several individual group differences that did emerge were not restricted to one hemisphere or the other or to a single brain area. Furthermore, the direction of the difference varied depending upon the cognitive condition and the brain region examined. These data indicate that state variables and testing conditions must be carefully considered and controlled in studies of lateralisation of brain function.

1 Geschwind N, Galaburda AM. Cerebral Lateralization. Cambridge: MIT Press, 1985.

2 Berker EA, Berker AH, Smith A. Translation of Broca's 1865 Report: Localization of speech in the third left frontal convolution. Arch Neurology 1986;43:1065-72.

3 Sperry RW. Hemispheric deconnection and unity in conscious awareness. Am Psychology 1968;23:723-33.

4 Gazzaniga MS. The Bisected Brain. New York: AppletonCentury Crofts, 1970

5 LeMay M, Kido DK. Asymmetries of the cerebral hemispheres on computed tomograms. Journal of Computer Assisted Tomography 1978;2:471-6.

6 Galaburda AM, LeMay M, Kemper TL, Geshwind N. Right-left asymmetries in the brain. Science 1978 199:852-6.

7 Kertesz A, Black SE, Polk M, Howell J. Cerebral asymmetries on magnetic resonance imaging. Cortex 1986; 22:117-27.

8 Gevins AS, Morgan NH, Bressler SL, et al. Human neuroelectric patterns predict performance accuracy. neuroelectric patterns
Science 1987;235:580-5.

9 Risberg J, Halsey JH, Wills EL, Wilson EM. Hemispheric specialization in normal man studied by bilateral measurements of the regional cerebral blood flow: a study with the 133-Xe inhalation technique. Brain 1975;98: with the

10 Gur RC, Gur RE, Obrist WK, et al. Sex and handedness differences in regional cerebral blood flow during rest and cognitive activity. Science 1982;217:659-61.

11 Wexler BE. Cerebral laterality and psychiatry: a review of the literature. Am J of Psychiatry 1980;137:279-91.

12 Gruzelier J, Flor-Henry P. Hemispheric asymmetries of function in psychopathology. New York: Elsevier North Holland Press, 1979.

13 Boucher J. Hand preference in autistic children and their parents. J Autism and Childhood Schizophrenia 1977;

14 Flor-Henry P, Yeudall L. Neuropsychological investigations of schizophrenia and manic-depressive psychosis. In: Gruzelier J, Flor-Henry P, eds. Hemispheric Asymmetries of Function in Psychopathology. New York: AsymNorth Holland Press, 1979:341-62.

15 Newlin DB, Carpenter B, Golden CJ. Hemispheric asymmetries in schizophrenia. Biological Psychiatry 1981; 16:561-81.

16 Nasrallah HA. Cerebral hemisphere asymmetries and interhemispheric integration in schizophrenia. In: Nasrallah HA, Weinberger DR, eds. Handbook of Schizo- 
phrenia, Vol 1: The Neurology of Schizophrenia. Amsterdam: Elsevier, 1986:157-74.

17 Andreasen NC, Grove W. The relationship between schizophrenic language, manic language, and aphasia. In: Gruzelier J, Flor-Henry P, eds. Hemispheric Asymmetries of Function in Psychopathology. New York: Elsevier North Holland Press, 1979:373-90.

18 Davison K, Bagley CR. Schizophrenia-like psychoses associated with organic disorders of the central nervous associated with organic disorders of the central nervous SP No 4:113-84.

19 Flor-Henry P. Psychosis and temporal lobe epilepsy: controlled investigation. Epilepsia 1969;10:363-95.

20 Gur RE. Left hemisphere dysfunction and left hemispheric overactivation in schizophrenia. Journal of Abnormal Psychology 1978;87:226-30.

21 Schweitzer L. Evidence of right cerebral hemisphere dysfunction in schizophrenic patients with left hemispheric overactivation. Biological Psychiatry 1982;17:655-73.

22 Beaumont JG, Dimond SJ. Brain disconnection and schizophrenia. Brit J Psychiatry 1973;123:661-2.

23 Green $P$. Defective interhemispheric transfer in schizophrenia. J Abnormal Psychology 1978;87:472-80.

24 Hartlage LG, Garber J. Spatial vs nonspatial reasoning ability in chronic schizophrenics. J Clin Psychology 1976;32:235-7.

25 Small JG, Sharpley PH, Milstein V, et al. Research diagnostic criteria and EEG findings in hospitalized schizophrenic patients. In: Obiols J, Gonzalezmonelus E, Pujo L, eds. Biological Psychiatry Today. Amsterdam: Elsevier North Holland Press, 1979.

26 Alpert M, Martz MJ. Cognitive views of schizophrenia in light of recent studies of brain asymmetry. In: Shagass $C$ Gershon S, Friedhoff AJ, eds. Psychopathology and brain dysfunctions. New York: Raven Press, 1977:1-13.

27 Lishman WA, Toone BK, Colburn CJ, McKeenan ERI Mance RM. Dichotic listening in psychotic patients. Brit J Psychiatry 1978;132:333-41.

28 Gruzelier JH. Bilateral asymmetry of skin conductance orienting activity and levels in schizophrenia. Biol Psychology 1973;1:21-41.

29 Gruzelier JH, Venables PH. Skin conductance orienting activity in a heterogeneous sample of schizophrenics. Nerv Ment Dis 1972;155:277-87.

30 Taylor MA, Greenspan B, Abrams R. Lateralized neuropsychological dysfunction in affective disorder and 1979:136:1031-4.

31 Taylor MA, Redfield J, Abrams R. Neuropsychologica dysfunction in schizophrenia and affective disease. Biological Psychiatry 1981;16:467-78

32 Seidman LJ. Schizophrenia and brain dysfunction: an integration of recent neurodiagnostic findings. Psycho Bull 1983;94:195-238.

33 Luchins DJ, Weinberger DR, Wyatt RJ. Cerebral asymmetry in schizophrenia determined by computed tomography. Am J Psychiatry 1982;139:753-7.

34 Shelton RC, Weinberger DR. X-ray computerized tomography studies in schizophrenia: a selective review and
synthesis.In: Nasrallah HA, Weinberger DR, eds. The Neurology of Schizophrenia. Amsterdam: Elsevier North Neurology of Schizophrenia.

35 Connolly JF, Gruzelier JH, Manchanda R, Hirsch SR. Visual evoked potentials in schizophrenia: Intensity effects and hemispheric asymmetry. Brit $J$ Psychiatry 983;142:152-5.

36 Flor-Henry P. Lateralized temporal-limbic dysfunction and psychopathology. Ann NY Acad Sci 1976;280:777-97.

37 Roemer RA, Shagass C, Straumanis JJ, Amadeo M. Patter evoked potential measurements suggesting lateralized hemispheric dysfunction in chronic schizophrenia. Biol Psychiatry 1978;13:185-202.

38 Buchsbaum MS, Carpenter WT, Fedio P, Goodwin FK Murphy DL, Post RM. Hemispheric differences in evoked potential enhancement by selective attention to hemiretinally presented stimuli in schizophrenic, affective and post-temporal lobectomy patients. In: Gruzelier J, Flor-Henry P, eds. Hemispheric Asymmetries of Function in Psychopathology. New York: Elsevier North Holland Psychopathology.

39 Mathew RJ, Duncan GC, Weiman ML, Barr DL. Regional cerebral blood flow in schizophrenia. Arch Gen Psychiatry cerebral blood flou

40 Gur RE, Skolnik BE, Gur RC, et al. Brain function in psychiatric disorders. I. Regional cerebral blood flow in medicated schizophrenics. Arch Gen Psychiatry 1983;40 $1250-4$

41 Gur RE, Gur RC, Skolnik BE, et al. Brain function in psychiatric disorders. III. Regional cerebral blood flow in unmedicated schizophrenics. Arch Gen Psychiatry 1985; 42:329-34.

42 Buchsbaum MS, Ingvar DH, Kessler R, et al. Cerebral glucography with positron tomography. Arch Gen Psychiatry 1982;39:251-9.

43 Sheppard G, Gruzelier J, Manchanda R, et al. 150-Positron emission tomography scanning in predominantly never treated acute schizophrenics. Lancet 1983;ii:1448-52.

44 Rumsey JM, Berman KF, Denckla MBV, et al. Regional cerebral blood flow in severe developmental dyslexia. Arch Neurology 1987;44:1144-50.

45 Obrist WD, Thompson HK, King HC, Wang HS. Determination of regional cerebral blood flow by inhalation of 133Xenon. Circ Res 1967;20:124-35.

46 Obrist WD, Thompson HK, Wang HS, Cronquist S. A simplified procedure for determining fast compartmen
rCBF by 133Xenon inhalation. In: Russell RWR, ed. Brain and Blood Flow. London: Pitman Publishing Company, 1971:11-5.

Wang HS, Wilkinson WE. Regional cerebral blood flow estimated by Xenon 133 inhalation. Stroke 1975;6:245-56.

48 Desmukh VD, Meyer JS. Noninvasive Measurement of Cerebral Blood Flow in Man. New York: Prentice Hall, 1978.

49 Risberg J. Regional cerebral blood flow measurements by 133Xe-inhalation: Methodology and application in neuropsychology and psychiatry. Brain and Language 1980;9:9-34.

50 Berman KF, Weinberger DR, Morihisa JM, Zec RF. Xenon 133 inhalation regional cerebral blood flow: Application to psychiatric research. In: Morihisa JM, ed. Brain Imaging in Psychiatry. Washington, DC: APA Press, 1984:42-64.

51 Weinberger DR, Berger KF, Zec RF. Physiological dysfunction of dorsolateral prefrontal cortex in schizophrenia: I. Regional cerebral blood flow (rCBF) evidence. Arch Gen Psychiatry 1986;43:114-25.

52 Berman KF, Weinberger DR. Cerebral blood flow studies in schizophrenia. In: Nasrallah $\mathrm{H}$, Weinberger DR, eds. The Neurology of Schizophrenia. Amsterdam: Elsevier North Holland Press, 1986:277-307.

53 Obrist WD, Wilkinson WE. The noninvasive Xe133 method: Evaluation of CBF indices. In: Bes A, Geraud G, eds. Cerebral Circulation. Amsterdam: Elsevier North Holland Inc, 1980:119-24.

54 McHenry LL, Merory J, Bass E, et al. Xenon-133 inhalation method for regional cerebral blood flow measurements: normal values and test-retest results. Stroke 1978;9:396-9.

55 Risberg J, Maximilian AV, Prohovnik I. Changes of cortical activity patterns during habituation to a reasoning test. Neuropsychologia 1977;15:793-8.

56 Berman KF, Zec RF, Weinberger DR. Physiological dysfunction of dorsolateral prefrontal cortex in schizophrenia: II. Role of neuroleptic treatment, attention, and mental effort. Arch Gen Psychiatry 1986;43:126-35.

57 Weinberger DR, Berman KF, Illowsky BP. Physiological dysfunction of dorsolateral prefrontal cortex in schizophrenia: III. A new cohort and evidence for a monoaminergic mechanism. Arch Gen Psychiatry 1988;45: $609-15$.

58 Berman KF, Illowsky BP, Weinberger DR. Physiological dysfunction of dorsolateral prefrontal cortex in schizophrenia. IV: Further evidence for regional and behavioural specificity. Arch Gen Psychiatry 1988;45 616-22.

59 Milner B. Effects of different brain lesions on card sorting Arch Neurology 1963;9:100-10.

60 Milner B. Some effects of frontal lobectomy in man. In: Warren JM, Akert K, eds. The Frontal Granular Cortex and Behavior. New York: McGraw-Hill, 1964:313-34.

61 Milner B. Interhemispheric differences in the localization of psychological processes in man. Br Med Bull 1971;27: $272-7$.

62 Grant AD, Berg EA A behavioral analysis of degree of reinforcement and ease of shifting to new responses in a Weigl-type card-sorting problem. J Exper Psychology

63 Rosvold HE, Mirsky AF, Sarason I, Bransome ED Jr, Beck LH. A continuous performance test of brain damage. $J$ Consul Clin Psychology 1956;20:343-50.

64 Raven JC. Progressive Matrices. London: HK Lewis and Co, 1938

65 Burke HR, Bingham WC. Raven's Progressive Matrices: More on construct validity. J Psychology 1969;72:247-51.

66 Reitan RM. Manual for administration of neuropsychological test batteries for adults and children. Tuscon: privately published by author, 1979 .

67 Benton AL, Hamsher DK, Varney NR, Spreen O. Contributions to Neuropsychological Assessment. New York: Oxford University Press, 1983.

68 Tominaga S, Strandgaard S, Uemura K, Ito K, Kutsuzawa $\mathrm{T}$. Cerebrovascular $\mathrm{CO} 2$ reactivity in normotensive and hypertensive man. Stroke 1976;7:507-10.

69 Maximilian VA, Prohovnik I, Risberg J. Cerebral hemodynamic responses to mental activation in normo and hyperapnia. Stroke 1980;11:342-7.

70 Davis SM, Ackerman RH, Correia JA, et al. Cerebral blood flow and cerebrovascular $\mathrm{CO} 2$ reactivity in stroke-age normal controls. Neurology 1983;33:391-9.

71 SAS Institute Inc. SAS User's Guide: Statistics, Version 5 Edition. Cary, NC: SAS Institute Inc, 1985.

72 Berman KF, Rosenbaum SCW, Brasher CA, Goldberg TE, Weinberger DR. Regional cerebral blood flow during auditory discrimination in schizophrenia. Abstracts Soc Neuroscience, 1987.

73 Prohovnik I, Epersen JO, Christensen FK. Development and initial evaluation of a heln

cerebrovascular disease. In Meyer JS, Shaw T, eds. Diagnosis and Management of Stroke. Menlo Park: Addison-Wesley Publishing Co, 1982.

75 Prohovnik I, Hakansson K, Risberg J. Observations on the functional significance of regional cerebral blood flow in resting normal subjects. Neuropsychologia 1980;18: resting $203-17$.

76 Ingvar DH, Cronquist S, Ekberg $\mathrm{K}$, Risberg J, HoedtRasmussen $\mathrm{K}$. Normal values of regional cerebral blood flow in man including flow and weight estimates of gray 
and white matter. Acta Neurol Scand 1965;41(S14):72-8. 77 Ingvar DH. Functional landscapes of the dominant hemisphere. Brain Research 1976;107:181-97.

78 Ingvar DH, Franzen G. Abnormalities of cerebral blood flow distribution in patients with chronic schizophrenia. Acta Psychiatrica Scandinavica 1974;50:425-62.

79 Roland PE. Focal increase of cerebral blood flow during stereognostic testing in man Arch Neurology 1976; 33:551-8.

80 Roland PE, Skinhoj E, Lasses NA. Focal alterations of human cerebral cortex during auditory discrimination. $J$ Neurophysiol 1981;45:1139-51.

81 Roland PE, Skinhoj E. Extrastriate cortical areas activated during visual discrimination in man. Brain Res 1981;222: during

82 Roland PE. Somatotopic tuning of postcentral gyrus during focal attention in man. A regional cerebral blood flow study. $J$ Neurophysiol $1981 ; 46: 744-54$

83 Duara R, Gross-Glenn K, Barker WW, et al. Behavioral activation and the variability of cerebral glucose metabolic measurements. J of Cerebral Blood Flow and Metabolism 1987;7:266-71.

84 Weinberger DR, Luchins DJ, Morihisa JM, Wyatt RJ. Asymmetric volumes of the right and left frontal and occipital regions of the human brain. Ann Neurology 1982; 11:97-100.

85 Kushner MJ, Rosenquist A, Alavi A, et al. Cerebral metabolism and patterned visual stimulation: a positron emis- sion tomography study of the human visual cortex. Neurology 1988;38:89-95.

86 Heilman KM, van den Abell T. Right hemisphere dominance for mediating cerebral activation. Neuropsychologia $1971 ; 17: 315-21$.

87 Heilman KM, van den Abell T. Right hemisphere dominance for attention: The mechanism underlying hemispheric asymmetries of inattention (neglect). Neurology 1980;30:327-30.

88 Mesulam MM. A cortical network for directed attention and unilateral neglect. Ann Neurology 1981;10:309-25.

89 Edfeldt AW. Silent Speech and Silent Reading. Chicago: University of Chicago Press, 1960.

90 Villardita C. Raven's colored progressive matrices and intellectual impairment in patients with focal brain damage. Cortex 1985;21:627-34.

91 Damasio AR, Damasio H, Chui HC. Neglect following damage to frontal lobe or basal ganglia. Neuropsychologic 1980;13:123-30.

92 Watson RT, Valenstein E, Heilman KM. Thalamic neglect: possible role of the medial thalamus and nucleus reticularis in behavior. Arch Neurology 1981;38:501-8.

93 Chapman LJ. Recent advances in the study of schizophrenic cognition. Schizophr Bull 1979;5:568-80.

94 Wohlberg GW, Kornetsky C. Sustained attention in remitted schizophrenics. Arch Gen Psychiatry 1973;28:533-7.

95 Orzack MH, Kornetsky C. Attention dysfunction in chronic schizophrenia. Arch Gen Psychiatry 1966;14:323-6. 